\title{
Monotonicity for excited random walk in high dimensions
}

\author{
Remco van der Hofstad • Mark Holmes
}

Received: 30 May 2008 / Revised: 16 March 2009 / Published online: 7 April 2009

(C) The Author(s) 2009. This article is published with open access at Springerlink.com

\begin{abstract}
We prove that the drift $\theta(d, \beta)$ for excited random walk in dimension $d$ is monotone in the excitement parameter $\beta \in[0,1]$, when $d$ is sufficiently large. We give an explicit criterion for monotonicity involving random walk Green's functions, and use rigorous numerical upper bounds provided by Hara (Private communication, 2007) to verify the criterion for $d \geq 9$.
\end{abstract}

Mathematics Subject Classification (2000) $\quad 60 \mathrm{~K} 35 \cdot 60 \mathrm{~K} 37$

\section{Introduction}

In this paper, we study excited random walk, where the random walker has a drift in the direction of the first component each time the walker visits a new site. It was shown that this process has ballistic behaviour when $d \geq 2$ in $[4,12,13]$. A nontrivial strong law of large numbers (SLLN) can then be obtained for $d \geq 2$ using renewal techniques (see for example $[14,16]$ ). For $d=1$, it is known that ERW is recurrent and diffusive [7] except in the trivial case $\beta=1$. Additional results on one-dimensional (multi)-excited random walks can be found in $[1-3,16]$.

In [15], a perturbative expansion was introduced and used to prove a weak law of large numbers and a central limit theorem for excited random walk in dimensions $d>5$

R. van der Hofstad $(\bowtie)$

Department of Mathematics and Computer Science, Eindhoven University of Technology,

P.O. Box 513, 5600 MB Eindhoven, The Netherlands

e-mail: rhofstad@win.tue.nl

M. Holmes

Department of Statistics, The University of Auckland,

Private Bag 92019, Auckland 1142, New Zealand

e-mail:mholmes@stat.auckland.ac.nz 
and $d>8$ respectively, with sufficiently small excitement parameter. More recently, [5] explicitly proved a SLLN and established a functional central limit theorem in dimensions $d \geq 2$. Included in [15] is an explicit representation of the drift in terms of the expansion coefficients. In this paper, we use this representation, together with explicit simple random walk Green's function bounds $[8,9]$ to prove that in dimensions $d \geq 9$, the drift for excited random walk is (strictly) increasing in the excitement parameter $\beta$.

\subsection{Main results}

The main result of this paper is the following theorem.

Theorem 1.1 (Monotonicity of the speed) For all $d \geq 9$, and $\beta \in[0,1]$, the drift for excited random walk in dimension d with excitement parameter $\beta$ is strictly increasing in $\beta$.

We are also able to show that for $d \geq 8$, there exists $\beta_{0}(d)$ such that the drift for ERW is strictly increasing in $\beta \in\left[0, \beta_{0}\right]$.

Simulations [5] suggest that the limiting variance of the first coordinate is not monotone in the excitement parameter $\beta$ in 2 dimensions. We expect that using the approach introduced in this paper, we can show that the variance is monotone decreasing in $\beta$ when the dimension is taken sufficiently high. By [15], the variance of the first coordinate is equal to $\sigma_{\beta}^{2} n(1+o(1))$ for some asymptotic variance $\sigma_{\beta}^{2}$, and based on our methods, we expect that $\sigma_{\beta}^{2}=d^{-1}-d^{-2} \beta^{2}+\beta^{2} \mathcal{O}\left(d^{-3}\right)$, showing that, in sufficiently high $d, \beta \mapsto \sigma_{\beta}^{2}$ is decreasing.

Although we only consider the once-excited random walk in this paper, the general multi-excited random walk can be handled with very minor modifications, yielding a result at least as strong as Theorem 1.1. A large part of the methodology in this paper can be applied more generally. See [11] for an application of the methods and results in this paper to the case where the drift on subsequent visits to a site is in the opposite direction to that induced by the excitement parameter on the first visit. Given the present context, another natural example is a random walk in an environment that is random in the first few coordinates only, with the expected drift induced by the environment denoted by $\vec{\beta}$. Some progress has been made in this direction [10] making use of the fact that a SLLN has been proved for general versions of such random walks in random environment in [6].

We first introduce some notation. A nearest-neighbour random walk path $\vec{\eta}$ is a sequence $\left\{\eta_{i}\right\}_{i=0}^{\infty}$ for which $\eta_{i} \in \mathbb{Z}^{d}$ and $\eta_{i+1}-\eta_{i}$ is a nearest-neighbour of the origin for all $i \geq 0$. For a general nearest-neighbour path $\vec{\eta}$ with $\eta_{0}=0$, we write $p^{\vec{\eta}_{i}}\left(x_{i}, x_{i+1}\right)$ for the conditional probability that the walk steps from $\eta_{i}=x_{i}$ to $x_{i+1}$, given the history of the path $\vec{\eta}_{i}=\left(\eta_{0}, \ldots, \eta_{i}\right)$. We write, for $\beta \in[0,1]$,

$$
p_{\beta}(x)=\frac{1+\beta e_{1} \cdot x}{2 d} I_{\{|x|=1\}},
$$


where $e_{1}=(1,0, \ldots, 0)$, and $x \cdot y$ is the inner-product between $x$ and $y$. Thus, $p_{\beta}$ is the transition probability for a random walk having a drift $\beta$ when stepping in the first coordinate. We write $\vec{\omega}_{n}$ for the $n$-step path of excited random walk (ERW), and $\mathbb{Q}$ for the law of $\left\{\vec{\omega}_{n}\right\}_{n=0}^{\infty}$, i.e., for every $n$-step nearest-neighbour path $\vec{\eta}_{n}$,

$$
\mathbb{Q}\left(\vec{\omega}_{n}=\vec{\eta}_{n}\right)=\prod_{i=0}^{n-1} p^{\vec{\eta}_{i}}\left(\eta_{i}, \eta_{i+1}\right)
$$

where $p^{0}\left(0, \eta_{1}\right)=p_{\beta}\left(\eta_{1}\right)$ is the probability to jump to $\eta_{1}$ in the first step, and

$$
p^{\vec{\eta}_{i}}\left(\eta_{i}, \eta_{i+1}\right)=p_{0}\left(\eta_{i+1}-\eta_{i}\right) I_{\left\{\eta_{i} \in \vec{\eta}_{i-1}\right\}}+p_{\beta}\left(\eta_{i+1}-\eta_{i}\right)\left[1-I_{\left\{\eta_{i} \in \vec{\eta}_{i-1}\right\}}\right],
$$

where $I_{\left\{\eta_{i} \in \vec{\eta}_{i-1}\right\}}$ denotes the indicator that $\eta_{i}=\eta_{j}$ for some $0 \leq j \leq i-1$. In words, the random walker gets excited each time he/she visits a new site, and when the random walk is excited, it has a positive drift in the direction of the first coordinate. For a description in terms of cookies, see [16].

\section{An overview of the proof and the expansion}

In this section, we recall some results and notation from [15]. If $\vec{\eta}$ and $\vec{\omega}$ are two paths of length at least $j$ and $m$ respectively and such that $\eta_{j}=\omega_{0}$, then the concatenation $\vec{\eta}_{j} \circ \vec{\omega}_{m}$ is defined by

$$
\left(\vec{\eta}_{j} \circ \vec{\omega}_{m}\right)_{i}= \begin{cases}\eta_{i} & \text { when } 0 \leq i \leq j \\ \omega_{i-j} & \text { when } j \leq i \leq m+j .\end{cases}
$$

Given $\vec{\eta}_{m}$ such that $\mathbb{Q}\left(\vec{\omega}_{m}=\vec{\eta}_{m}\right)>0$, we define a probability measure $\mathbb{Q}^{\vec{\eta}_{m}}$ on paths starting from $\eta_{m}$ by specifying its value on particular cylinder sets (in a consistent manner) as follows

$$
\mathbb{Q}^{\vec{\eta}_{m}}\left(\vec{\omega}_{n}=\vec{\mu}_{n}\right) \equiv \prod_{i=0}^{n-1} p^{\vec{\eta}_{m} \circ \vec{\mu}_{i}}\left(\mu_{i}, \mu_{i+1}\right)
$$

and extending the measure to all finite-dimensional cylinder sets in the natural (consistent) way. Then (2.2) is also $\mathbb{Q}\left(\vec{\omega}_{m+n}=\vec{\eta}_{m} \circ \vec{\mu}_{n} \mid \vec{\omega}_{m}=\vec{\eta}_{m}\right)$.

In [15], a perturbative expansion was derived for the two-point function $c_{n}(x)=$ $\mathbb{Q}\left(\omega_{n}=x\right)$, giving rise to a recursion relation of the form

$$
c_{n+1}(x)=\sum_{y \in \mathbb{Z}^{d}} p_{\beta}(y) c_{n}(x-y)+\sum_{m=2}^{n+1} \sum_{y \in \mathbb{Z}^{d}} \pi_{m}(y) c_{n+1-m}(x-y) .
$$

This expansion was used to prove a law of large numbers and central limit theorem for ERW. We next discuss the coefficients $\pi_{m}(y)$ and some results of this expansion. 
The expansion coefficients Let $N \geq 1$, and for $i \geq 0$, let $\vec{\omega}_{j_{i}+1}^{(i)}$ be a path of length $j_{i}+1 \in \mathbb{Z}_{+}$, where, by convention, $j_{0}=0$. Then define

$$
\Delta_{N}=\left(p^{\vec{\omega}_{j_{N-1}+1}^{(N-1)} \circ \vec{\omega}_{j_{N}}^{(N)}}-p^{\vec{\omega}_{j_{N}}^{(N)}}\right)\left(\omega_{j_{N}}^{(N)}, \omega_{j_{N}+1}^{(N)}\right)
$$

which depends on $\vec{\omega}_{j_{N-1}+1}^{(N-1)}$ and $\vec{\omega}_{j_{N}+1}^{(N)}$ (although this dependence is suppressed in the notation). The difference (2.4) is identically zero when the histories $\vec{\omega}_{j_{N-1}+1}^{(N-1)} \circ \vec{\omega}_{j_{N}}^{(N)}$ and $\vec{\omega}_{j_{N}}^{(N)}$ give the same transition probabilities to go from $\omega_{j_{N}}^{(N)}$ to $\omega_{j_{N}+1}^{(N)}$. For excited random walk, $\Delta_{N}$ is non-zero precisely when $\omega_{j_{N}}^{(N)}$ has already been visited by $\vec{\omega}_{j_{N-1}+1}^{(N-1)}$ but not by $\vec{\omega}_{j_{N}-1}^{(N)}$, so that

$$
\begin{aligned}
\left|\Delta_{N}\right| & =\left|\frac{\beta e_{1} \cdot\left(\omega_{j_{N}+1}^{(N)}-\omega_{j_{N}}^{(N)}\right)}{2 d}\left[I_{\left\{\omega_{j_{N}}^{(N)} \notin \vec{\omega}_{j_{N-1}}^{(N-1)} \circ \vec{\omega}_{j_{N}-1}^{(N)}\right\}}-I_{\left\{\omega_{j_{N}}^{(N)} \notin \vec{\omega}_{j_{N}-1}^{(N)}\right\}}\right]\right| \\
& \leq \frac{\beta}{2 d} I_{\left\{\omega_{j_{N}+1}^{(N)}=\omega_{j_{N}}^{(N)} \pm e_{1}\right\}} I_{\left\{\omega_{j_{N}}^{(N)} \in \vec{\omega}_{j_{N-1}}^{(N-1)} \backslash \vec{\omega}_{j_{N}-1}^{(N)}\right\}} \leq \frac{\beta}{2 d} I_{\left\{\omega_{j_{N}+1}^{(N)}=\omega_{j_{N}}^{(N)} \pm e_{1}\right\}} I_{\left\{\omega_{j_{N}}^{(N)} \in \vec{\omega}_{j_{N-1}}^{(N-1)}\right\}} .
\end{aligned}
$$

Define $\mathcal{A}_{m, N}=\left\{\left(j_{1}, \ldots, j_{N}\right) \in \mathbb{Z}_{+}^{N}: \sum_{l=1}^{N} j_{l}=m-N-1\right\}, \mathcal{A}_{N}=\dot{\cup}_{m} \mathcal{A}_{m, N}$ and

$$
\begin{aligned}
\pi_{m}^{(N)}(x, y)= & \sum_{\vec{j} \in \mathcal{A}_{m, N}} \sum_{\vec{\omega}_{1}^{(0)}} \sum_{\vec{\omega}_{j_{1}+1}^{(1)}} \cdots \sum_{\vec{\omega}_{j_{N}+1}^{(N)}} I_{\left\{\omega_{j_{N}}^{(N)}=x, \omega_{j_{N}+1}^{(N)}=y\right\}} p_{\beta}\left(\omega_{1}^{(0)}\right) \\
& \times \prod_{n=1}^{N} \Delta_{n} \prod_{i_{n}=0}^{j_{n}-1} p^{\vec{\omega}_{j_{n-1}+1}^{(n-1)} \circ \vec{\omega}_{i_{n}}^{(n)}}\left(\omega_{i_{n}}^{(n)}, \omega_{i_{n}+1}^{(n)}\right) .
\end{aligned}
$$

Then we define

$$
\begin{aligned}
\pi_{m}(x, y) & =\sum_{N=1}^{\infty} \pi_{m}^{(N)}(x, y), \quad \pi^{(N)}(x, y)=\sum_{m} \pi_{m}^{(N)}(x, y), \quad \text { and } \\
\pi_{m}(y) & =\sum_{N=1}^{\infty} \sum_{x \in \mathbb{Z}^{d}} \pi_{m}^{(N)}(x, y) .
\end{aligned}
$$

Note that the quantities $\pi_{m}^{(N)}$ are all zero when $N+1>m$, and that all of the above quantities depend on $\beta$. Note further that

$$
\sum_{y \in \mathbb{Z}^{d}} \pi_{m}^{(N)}(x, y)=0
$$

since $\sum_{\omega_{j_{N}+1}^{(N)}} \Delta_{N}=0($ see also $[15,(6.10)])$. 
The importance of these quantities is given by [15, Proposition 3.1], which states that if $\lim _{n \rightarrow \infty} \sum_{m=2}^{n} \sum_{x \in \mathbb{Z}^{d}} x \pi_{m}(x)$ exists and $n^{-1} \omega_{n}$ converges in probability to $\theta$, then

$$
\theta(\beta, d)=\sum_{y \in \mathbb{Z}^{d}} y p_{\beta}(y)+\sum_{m=2}^{\infty} \sum_{y \in \mathbb{Z}^{d}} y \pi_{m}(y)=\frac{\beta e_{1}}{d}+\sum_{m=2}^{\infty} \sum_{y \in \mathbb{Z}^{d}} y \pi_{m}(y)
$$

Strategy of the proof of Theorem 1.1 We shall explicitly differentiate the right hand side of (2.9), and prove that this derivative is positive for all $\beta \in[0,1]$, when $d \geq 9$. From (2.9) and using (2.7) and (2.8), we have

$$
\sum_{y \in \mathbb{Z}^{d}} y \pi_{m}(y)=\sum_{x, y \in \mathbb{Z}^{d}}(y-x) \pi_{m}(x, y),
$$

so that

$$
\theta(\beta, d)=\frac{\beta e_{1}}{d}+\sum_{m=2}^{\infty} \sum_{N=1}^{\infty} \sum_{x, y \in \mathbb{Z}^{d}}(y-x) \pi_{m}^{(N)}(x, y)
$$

Letting $\varphi_{m}^{(N)}(x, y)=\frac{\partial}{\partial \beta} \pi_{m}^{(N)}(x, y)$ and assuming that the limit can be taken through the infinite sums, we then have

$$
\frac{\partial \theta}{\partial \beta}(\beta, d)=\frac{e_{1}}{d}+\sum_{N=1}^{\infty} \sum_{m=2}^{\infty} \sum_{x, y \in \mathbb{Z}^{d}}(y-x) \varphi_{m}^{(N)}(x, y)
$$

Since $\varphi_{m}^{(N)}(x, y) \equiv 0$ unless $|x-y|=1$, we have that

$$
\left|\frac{\partial \theta}{\partial \beta}(\beta, d)-\frac{e_{1}}{d}\right| \leq \sum_{N=1}^{\infty} \sum_{m=2}^{\infty} \sum_{x, y \in \mathbb{Z}^{d}}\left|\varphi_{m}^{(N)}(x, y)\right| .
$$

We conclude that $\frac{\partial \theta_{1}}{\partial \beta}(\beta, d)$, which is the first coordinate of $\frac{\partial \theta}{\partial \beta}(\beta, d)$, is positive for any $\beta$ at which $\sum_{N=1}^{\infty} \sum_{m=2}^{\infty} \sum_{x, y \in \mathbb{Z}^{d}}\left|\varphi_{m}^{(N)}(x, y)\right|<d^{-1}$. This is what we shall prove in the remainder of this paper, which is organised as follows. In Sect. 3, we start by proving bounds on $\pi_{m}^{(N)}$. These bounds will be crucially used to prove bounds on $\varphi_{m}^{(N)}$ in Sect. 4. The results in Sect. 4 are used in Sect. 5 to prove Theorem 1.1.

\section{Bound on $\pi$}

Before proceeding to the proof of Theorem 1.1, we prove a new bound on $\sum_{x, y \in \mathbb{Z}^{d}} \sum_{m}\left|\pi_{m}^{(N)}(x, y)\right|$. The proof of this new bound makes use of Lemma 3.1 below. 
Let $\mathbb{P}_{d}$ denote the law of simple symmetric random walk in $d$ dimensions, starting at the origin, and let $D_{d}(x)=I_{\{|x|=1\}} /(2 d)$ be the simple random walk step distribution. We will make use of the convolution of functions, which is defined for absolutely summable functions $f, g$ on $\mathbb{Z}^{d}$ by

$$
(f * g)(x)=\sum_{y \in \mathbb{Z}^{d}} f(y) g(x-y)
$$

Let $f^{* k}$ denote the $k$-fold convolution of $f$ with itself, and let $G_{d}(x)=\sum_{k=0}^{\infty} D_{d}^{* k}(x)$ denote the Green's function for this random walk. We shall sometimes make use of the representation

$$
\begin{aligned}
G_{d}^{* i}(x) & =\sum_{k=0}^{\infty} \sum_{\vec{m}_{i}: m_{1}+\cdots+m_{i}=k} D_{d}^{*\left(m_{1}+\cdots+m_{i}\right)}(x) \\
& =\sum_{k=0}^{\infty} \frac{(k+i-1) !}{(i-1) ! k !} \mathbb{P}_{d}\left(\omega_{k}=x\right), \quad \text { for } i \geq 1 .
\end{aligned}
$$

Note that $G_{d}^{* i}(x)<\infty$ if and only if $d>2 i$. We shall often abbreviate $G_{d}^{* i}=G_{d}^{* i}(0)$. For $i \geq 0$, let

$$
\mathcal{E}_{i}(d)=\sup _{v \in \mathbb{Z}^{d-1}}\left(\left(\frac{d}{d-1}\right)^{i+1} G_{d-1}^{*(i+1)}(v)-\delta_{0, v}\right)
$$

Lemma 3.1 (Diagrammatic bounds for ERW) For excited random walk, uniformly in $u \in \mathbb{Z}^{d}$ and $\vec{\eta}_{m}$, for $i \geq 0$,

$$
\begin{aligned}
& \sum_{j=0}^{\infty} \frac{(j+i) !}{j !} \mathbb{Q}^{\vec{\eta}_{m}}\left(\omega_{j}=u\right) \leq i !\left(\frac{d}{d-1}\right)^{i+1} G_{d-1}^{*(i+1)} \\
& \sum_{j=1}^{\infty} \frac{(j+i) !}{j !} \mathbb{Q}^{\vec{\eta}_{m}}\left(\omega_{j}=u\right) \leq i ! \mathcal{E}_{i}(d) .
\end{aligned}
$$

Proof Define an increasing sequence of random variables $\mathcal{N}_{j}, j \geq 0$, by letting $j-\mathcal{N}_{j}$ be the number of steps that the walk $\vec{\omega}_{j}$ takes in the first coordinate. Observe that independently of $\vec{\eta}, \mathcal{N}_{j}$ has a $\operatorname{Binomial}\left(j, q_{d}\right)$ distribution, where $q_{d}=(d-1) / d$. If we consider $\vec{\omega}_{j}$ as the initial position and first $j$ steps of an infinite walk $\vec{\omega}$, then the sequence $\left\{\mathcal{N}_{j}\right\}_{j \geq 0}$ is a random walk on $\mathbb{Z}_{+}$taking i.i.d. steps that are either +1 or 0 with probability $q_{d}$ and $1-q_{d}$ respectively. The random time that such a walk spends at any level $l$ has a Geometric distribution with parameter $q_{d}$. Thus, writing $\mathcal{P}$ for the 
law of $\left\{\mathcal{N}_{j}\right\}_{j=0}^{\infty}$, we obtain that for every $i \geq 0$,

$$
\begin{aligned}
\frac{(j+i) !}{j !} \mathcal{P}\left(\mathcal{N}_{j}=l\right) & =\frac{(j+i) !}{j !} \frac{j !}{l !(j-l) !} q_{d}^{l}\left(1-q_{d}\right)^{j-l} \\
& =q_{d}^{-i} \frac{(l+i) !}{l !} \mathcal{P}\left(\mathcal{N}_{j+i}=l+i\right)
\end{aligned}
$$

so that, for $m \leq l$,

$$
\sum_{j=m}^{\infty} \frac{(j+i) !}{j !} \mathcal{P}\left(\mathcal{N}_{j}=l\right)=q_{d}^{-(i+1)} \frac{(l+i) !}{l !}
$$

Given $u=\left(u_{1}, \ldots, u_{d}\right) \in \mathbb{Z}^{d}$, we write $u^{-}:=\left(u_{2}, u_{3}, \ldots, u_{d}\right) \in \mathbb{Z}^{d-1}$. To prove (3.4), note that

$$
\begin{aligned}
\sum_{j=0}^{\infty} \frac{(j+i) !}{j !} \mathbb{Q}^{\vec{\eta}_{m}}\left(\omega_{j}=u\right) & =\sum_{j=0}^{\infty} \frac{(j+i) !}{j !} \sum_{l=0}^{j} \mathbb{Q}^{\vec{\eta}_{m}}\left(\omega_{j}=u \mid \mathcal{N}_{j}=l\right) \mathcal{P}\left(\mathcal{N}_{j}=l\right) \\
& \leq \sum_{l=0}^{\infty} \mathbb{P}_{d-1}\left(\omega_{l}=u^{-}-\eta_{m}^{-}\right) \sum_{j=l}^{\infty} \frac{(j+i) !}{j !} \mathcal{P}\left(\mathcal{N}_{j}=l\right) \\
& \leq q_{d}^{-(i+1)} \sup _{v \in \mathbb{Z}^{d-1}} \sum_{l=0}^{\infty} \mathbb{P}_{d-1}\left(\omega_{l}=v\right) \frac{(l+i) !}{l !}
\end{aligned}
$$

By (3.2), (3.7) is equal to $i ! q_{d}^{-(i+1)} \sup _{v \in \mathbb{Z}^{d-1}} G_{d-1}^{*(i+1)}(v)$. By [9, Lemma B.3], the supremum occurs at $v=0$. Since $q_{d}^{-1}=d /(d-1)$, this proves (3.4).

The bound (3.5) is proved similarly. Indeed, for $i \geq 0$, we can write

$$
\begin{aligned}
& \sum_{j=1}^{\infty} \frac{(j+i) !}{j !} \mathbb{Q}^{\vec{\eta}_{m}}\left(\omega_{j}=u\right) \leq \sup _{v \in \mathbb{Z}^{d-1}} \sum_{l=0}^{\infty} \mathbb{P}_{d-1}\left(\omega_{l}=v\right) \sum_{j=l \vee 1}^{\infty} \frac{(j+i) !}{j !} \mathcal{P}\left(\mathcal{N}_{j}=l\right) \\
& =\sup _{v \in \mathbb{Z}^{d-1}}\left(\sum_{l=0}^{\infty} \mathbb{P}_{d-1}\left(\omega_{l}=v\right)\left[\sum_{j=l}^{\infty} \frac{(j+i) !}{j !} \mathcal{P}\left(\mathcal{N}_{j}=l\right)-\delta_{0, l} i ! \mathcal{P}\left(\mathcal{N}_{0}=0\right)\right]\right) \\
& =\sup _{v \in \mathbb{Z}^{d-1}}\left(q_{d}^{-(i+1)} \sum_{l=0}^{\infty} \frac{(l+i) !}{l !} \mathbb{P}_{d-1}\left(\omega_{l}=v\right)-i ! \delta_{0, v}\right) \\
& =i ! \sup _{v \in \mathbb{Z}^{d-1}}\left(q_{d}^{-(i+1)} G_{d-1}^{*(i+1)}(v)-\delta_{0, v}\right),
\end{aligned}
$$

since $\mathcal{P}\left(\mathcal{N}_{0}=0\right)=1$ and $\sum_{l=0}^{\infty} \mathbb{P}_{d-1}\left(\omega_{l}=v\right) \delta_{0, l}=\delta_{0, v}$, and following the steps in (3.7) above. 
Define

$$
a_{d}=\frac{d}{(d-1)^{2}} G_{d-1}^{* 2}
$$

Proposition 3.2 (Bounds on the expansion coefficients for ERW) For $N=1$, $\sum_{x, y \in \mathbb{Z}^{d}} \sum_{m}\left|\pi_{m}^{(1)}(x, y)\right| \leq \beta d^{-1} \mathcal{E}_{0}(d)$, and, for $N \geq 2$,

$$
\sum_{x, y \in \mathbb{Z}^{d}} \sum_{m}\left|\pi_{m}^{(N)}(x, y)\right| \leq \beta^{N} d^{-1}(d-1)^{-1} G_{d-1} \mathcal{E}_{1}(d) a_{d}^{N-2} .
$$

Given $\vec{\eta}_{m}$ and $\vec{z}_{j+1}$, define

$$
\Delta\left(\vec{z}_{j+1}\right)=\left(p^{\vec{\eta}_{m} \circ \vec{z}_{j}}\left(z_{j}, z_{j+1}\right)-p^{\vec{z}_{j}}\left(z_{j}, z_{j+1}\right)\right) I_{\left\{z_{0}=\eta_{m}\right\}} \cdot
$$

We will use the following lemma to prove Proposition 3.2.

Lemma 3.3 (Ingredients for bounds on lace expansion coefficients) For any $\vec{\eta}_{s}$,

$$
\begin{aligned}
& \sum_{j=0}^{\infty} \sum_{\vec{z}_{j+1}}\left|\Delta\left(\vec{z}_{j+1}\right)\right| \prod_{i=0}^{j-1} p^{\vec{\eta}_{s} \circ \vec{z}_{i}}\left(z_{i}, z_{i+1}\right) \leq s \beta \frac{G_{d-1}}{d-1}, \\
& \sum_{j=0}^{\infty}(j+1) \sum_{\vec{z}_{j+1}}\left|\Delta\left(\vec{z}_{j+1}\right)\right| \prod_{i=0}^{j-1} p^{\vec{\eta}_{s} \circ \vec{z}_{i}}\left(z_{i}, z_{i+1}\right) \leq s \beta a_{d}, \\
& \sum_{j=1}^{\infty} \sum_{\vec{z}_{j+1}}\left|\Delta\left(\vec{z}_{j+1}\right)\right| \prod_{i=0}^{j-1} p^{\vec{\eta}_{s} \circ \vec{z}_{i}}\left(z_{i}, z_{i+1}\right) \leq s \beta \frac{\mathcal{E}_{0}(d)}{d}, \\
& \sum_{j=1}^{\infty}(j+1) \sum_{\vec{z}_{j+1}}\left|\Delta\left(\vec{z}_{j+1}\right)\right| \prod_{i=0}^{j-1} p^{\vec{\eta}_{s} \circ \vec{z}_{i}}\left(z_{i}, z_{i+1}\right) \leq s \beta \frac{\mathcal{E}_{1}(d)}{d} .
\end{aligned}
$$

Proof As in (2.5), the left hand side of (3.12) is bounded above by

$$
\begin{gathered}
\sum_{j=0}^{\infty} \sum_{\vec{z}_{j}}\left(\prod_{i=0}^{j-1} p^{\vec{\eta}_{s} \circ \vec{z}_{i}}\left(z_{i}, z_{i+1}\right)\right) I_{\left\{z_{j} \in \vec{\eta}_{s-1}\right\}} \frac{\beta}{2 d} \sum_{z_{j+1}} I_{\left\{z_{j+1}=z_{j} \pm e_{1}\right\}} \\
\leq \frac{\beta}{d} \sum_{j=0}^{\infty} \sum_{\vec{z}_{j}}\left(\prod_{i=0}^{j-1} p^{\vec{\eta}_{s} \circ \vec{z}_{i}}\left(z_{i}, z_{i+1}\right)\right) I_{\left\{z_{j} \in \vec{\eta}_{s-1}\right\}},
\end{gathered}
$$

since only two terms contribute to the rightmost sum in the first line of (3.16). From (2.2), this is equal to 


$$
\begin{aligned}
\frac{\beta}{d} \sum_{j=0}^{\infty} \sum_{\vec{z}_{j}} \mathbb{Q}^{\vec{\eta}_{s}}\left(\vec{\omega}_{j}=\vec{z}_{j}\right) I_{\left\{z_{j} \in \vec{\eta}_{s-1}\right\}} & =\frac{\beta}{d} \sum_{j=0}^{\infty} \mathbb{Q}^{\vec{\eta}_{s}}\left(\omega_{j} \in \vec{\eta}_{s-1}\right) \\
& \leq \frac{\beta}{d} \sum_{l=0}^{s-1} \sum_{j=0}^{\infty} \mathbb{Q}^{\vec{\eta}_{s}}\left(\omega_{j}=\eta_{l}\right) .
\end{aligned}
$$

The inequality (3.12) then follows from (3.4) with $i=0$. The inequality (3.13) is obtained similarly, using (3.4) with $i=1$ at the last step, while (3.14) and (3.15) are obtained using (3.5) with $i=0$ and $i=1$ respectively at the last step.

Proof of Proposition 3.2 It follows from (2.6) that $\sum_{x, y \in \mathbb{Z}^{d}} \sum_{m}\left|\pi_{m}^{(N)}(x, y)\right|$ is bounded by

$$
\begin{gathered}
\sum_{\omega_{1}^{(0)}} p_{\beta}\left(\omega_{1}^{(0)}\right) \sum_{j_{1}=1}^{\infty} \sum_{\vec{\omega}_{j_{1}+1}^{(1)}}\left|\Delta_{1}\right| \prod_{i_{1}=1}^{j_{1}-1} p^{\vec{\omega}_{1}^{(0)} \circ \vec{\omega}_{i_{1}}^{(1)}}\left(\omega_{i_{1}}^{(1)}, \omega_{i_{1}+1}^{(1)}\right) \\
\quad \ldots \sum_{j_{N}=0}^{\infty} \sum_{\vec{\omega}_{j_{N}+1}^{(N)}}\left|\Delta_{N}\right| \prod_{i_{N}=1}^{j_{N}-1} p^{\vec{\omega}_{j_{N-1}+1}^{(N-1)} \circ \vec{\omega}_{i_{N}}^{(N)}}\left(\omega_{i_{N}}^{(N)}, \omega_{i_{N}+1}^{(N)}\right),
\end{gathered}
$$

where the sums over $j_{k}, k \geq 2$ are all from 0 to $\infty$. Note that $\Delta_{1}$ can only be non-zero if $j_{1}$ is odd (so in particular, non-zero). We proceed by using Lemma 3.3 to successively bound the sums over $j_{k}$ of this expression, beginning with the sum over $j_{N}$.

If $N=1$ then we use (3.14) with $s=1$ to bound this sum by $s \beta \frac{\mathcal{E}_{0}(d)}{d}$, and then $\sum_{\omega_{1}^{(0)}} p_{\beta}\left(\omega_{1}^{(0)}\right)=1$ gives the result. If $N>1$ then we use (3.12) with $s=j_{N-1}+1$ on the sum over $j_{N}$, followed by repeated applications of (3.13) with $s=j_{k-1}+1$ on the sums over $j_{k}$ with $k=N-1, \ldots, 2$ respectively, then (3.15) with $s=1$ on the sum over $j_{1}$ and again the result follows since $\sum_{\omega_{1}^{(0)}} p_{\beta}\left(\omega_{1}^{(0)}\right)=1$.

Since the speed is known to exist [5], the following corollary is an easy consequence of [15, Propositions 3.1 and 6.1] together with Proposition 3.2, and the fact that $a_{6}<1$ since $G_{5}^{* 2}<5^{2} / 6[9]$.

Corollary 3.4 (Formula for the speed of ERW) For all $d \geq 6$ and $\beta \in[0,1]$,

$$
\theta(\beta, d)=\lim _{n \rightarrow \infty} \mathbb{E}\left[\omega_{n+1}-\omega_{n}\right]=\frac{\beta e_{1}}{d}+\sum_{m=2}^{\infty} \sum_{x \in \mathbb{Z}^{d}} x \pi_{m}(x)
$$

In fact, the first equality in Corollary 3.4 holds for all $d \geq 2$ since the law $\mu_{n}$ of the cookie environment as viewed by the random walker at time $n$ is known to converge 
(see e.g. [5]) and

$$
\begin{aligned}
\mathbb{E}\left[\omega_{n+1}-\omega_{n}\right] & =\mathbb{E}\left[\mathbb{E}\left[\omega_{n+1}-\omega_{n} \mid \vec{\omega}_{n}\right]\right]=\mathbb{E}\left[\frac{\beta e_{1}}{d} I_{\left\{\omega_{n} \notin \vec{\omega}_{n-1}\right\}}\right] \\
& =\frac{\beta e_{1}}{d}\left[1-\mathbb{P}\left(\omega_{n} \in \vec{\omega}_{n-1}\right)\right],
\end{aligned}
$$

where the right hand side which converges as $n \rightarrow \infty$ since $\mathbb{P}\left(\omega_{n} \in \vec{\omega}_{n-1}\right)$ is the $\mu_{n}$-measure of the event that the cookie at the origin is absent.

\section{The differentiation step}

To verify the exchange of limits in (2.12), it is sufficient to prove that $\sum_{x, y \in \mathbb{Z}^{d}}(y-x)$ $\pi_{m}^{(N)}(x, y)$ is absolutely summable in $m$ and $N$ (note that for every $m$ and $N$ the summations over $x$ and $y$ are finite) and that $\sum_{N=1}^{\infty} \sum_{m=2}^{\infty} \sup _{\beta \in[0,1]} \mid \sum_{x, y \in \mathbb{Z}^{d}}(y-x)$ $\varphi_{m}^{(N)}(x, y) \mid<\infty$. By Proposition 3.2 and the fact that $|y-x|=1$ for $x, y$ nearest neighbours, the first condition holds provided that

$$
\beta a_{d}<1
$$

In fact we will see later on that this inequality for $\beta=1$ is sufficient to also establish the second condition. We now identify $\varphi_{m}^{(N)}(x, y)$.

Recall (2.6). Then we can write

$$
\varphi_{m}^{(N)}(x, y)=\varphi_{m}^{(N, 1)}(x, y)+\varphi_{m}^{(N, 2)}(x, y)+\varphi_{m}^{(N, 3)}(x, y)
$$

where (by Leibniz' rule), $\varphi_{m}^{(N, 1)}(x, y), \varphi_{m}^{(N, 2)}(x, y)$ and $\varphi_{m}^{(N, 3)}(x, y)$ arise from differentiating $p_{\beta}\left(\omega_{1}^{(0)}\right), \prod_{n=1}^{N} \prod_{i_{n}=0}^{j_{n}-1} p^{\vec{\omega}_{j_{n-1}+1}^{(n-1)} \circ \vec{\omega}_{i_{n}}^{(n)}}\left(\omega_{i_{n}}^{(n)}, \omega_{i_{n}+1}^{(n)}\right)$ and $\prod_{n=1}^{N} \Delta_{n}$, respectively, with respect to $\beta$.

Observe that if $\eta_{m}=x_{l}$ then

$$
\begin{aligned}
\frac{\partial}{\partial \beta} p_{\beta}^{\vec{\eta}_{m}}\left(x_{l}, x\right) & =\frac{e_{1} \cdot\left(x-x_{l}\right) I_{\left\{x_{l} \notin \vec{\eta}_{m-1}\right\}}}{2 d} I_{\left\{\left|x-x_{l}\right|=1\right\}} \\
& =\frac{I_{\left\{x_{l} \notin \vec{\eta}_{m-1}\right\}}}{2 d}\left(I_{\left\{x-x_{l}=e_{1}\right\}}-I_{\left\{x-x_{l}=-e_{1}\right\}}\right),
\end{aligned}
$$

and hence, using $I_{A}-I_{A \cap C}=I_{A \cap C^{c}}$ we have

$$
\frac{\partial}{\partial \beta}\left(p_{\beta}^{\vec{\eta}_{m}}\left(x_{l}, x\right)-p_{\beta}^{\vec{\omega}_{n} \circ \vec{\eta}_{m}}\left(x_{l}, x\right)\right)=\frac{1}{2 d} I_{\left\{x_{l} \notin \vec{\eta}_{m-1}, x_{l} \in \vec{\omega}_{n-1}\right\}}\left(I_{\left\{x-x_{l}=e_{1}\right\}}-I_{\left\{x-x_{l}=-e_{1}\right\}}\right) .
$$


Clearly then

$$
\left|\frac{\partial}{\partial \beta}\left(p_{\beta}^{\vec{\eta}_{m}}\left(x_{l}, x\right)-p_{\beta}^{\vec{\omega}_{n} \circ \vec{\eta}_{m}}\left(x_{l}, x\right)\right)\right| \leq \frac{1}{2 d} I_{\left\{x_{l} \in \vec{\omega}_{n-1} \backslash \vec{\eta}_{m-1}\right\}}\left(I_{\left\{x-x_{l}=e_{1}\right\}}+I_{\left\{x-x_{l}=-e_{1}\right\}}\right) .
$$

Let $\rho^{(N)}$ be the quantity obtained by replacing $p_{\beta}\left(\omega_{1}^{(0)}\right)$ in (2.6) with $(2 d)^{-1}$ $I_{\left\{\omega_{1}^{(0)}= \pm e_{1}\right\}}$ (a bound on its derivative) and by bounding $\Delta_{n}$ by $\left|\Delta_{n}\right|$ for all $n=1, \ldots, N$.

For $k=1, \ldots, N$, let $\gamma_{k}^{(N)}$ be the quantity obtained from (2.6) by bounding $\Delta_{n}$ by $\left|\Delta_{n}\right|$ for all $n=1, \ldots, N$ and by replacing $\prod_{i_{k}=0}^{j_{k}-1} p^{\vec{\omega}_{j_{k-1}+1}^{(k-1)} \circ \vec{\omega}_{i_{k}}^{(k)}}\left(\omega_{i_{k}}^{(k)}, \omega_{i_{k}+1}^{(k)}\right)$ with the following bound on its derivative

$$
\sum_{l=0}^{j_{k}-1} \frac{I_{\left\{\omega_{l+1}^{(k)}-\omega_{l}^{(k)}= \pm e_{1}\right\}}}{2 d} \prod_{\substack{i_{k}=0 \\ i_{k} \neq l}}^{j_{k}-1} p^{\vec{\omega}_{j_{k-1}+1}^{(k-1)} \circ \vec{\omega}_{i_{k}}^{(k)}}\left(\omega_{i_{k}}^{(k)}, \omega_{i_{k}+1}^{(k)}\right) .
$$

Similarly, let $\chi_{k}^{(N)}$ be obtained by replacing $\Delta_{k}$ in (2.6) by $(2 d)^{-1} I_{\left\{\omega_{j_{k}}^{(k)} \in \vec{\omega}_{j_{k-1}}^{(k-1)}\right\}}$ $I_{\left\{\omega_{j_{k}+1}^{(k)}-\omega_{j_{k}}^{(k)}= \pm e_{1}\right\}}$ (a bound on its derivative) and by bounding $\Delta_{n}$ for $n \neq k$ by $\left|\Delta_{n}\right|$.

Letting $\gamma^{(N)}=\sum_{k=1}^{N} \gamma_{k}^{(N)}$ and $\chi^{(N)}=\sum_{k=1}^{N} \chi_{k}^{(N)}$, we obtain

$$
\begin{aligned}
& \sum_{m} \sum_{x, y \in \mathbb{Z}^{d}}\left|\varphi_{m}^{(N, 1)}(x, y)\right| \leq \rho^{(N)}, \quad \sum_{m} \sum_{x, y \in \mathbb{Z}^{d}}\left|\varphi_{m}^{(N, 2)}(x, y)\right| \leq \gamma^{(N)}, \quad \text { and } \\
& \sum_{m} \sum_{x, y \in \mathbb{Z}^{d}}\left|\varphi_{m}^{(N, 3)}(x, y)\right| \leq \chi^{(N)} .
\end{aligned}
$$

We shall bound each of these terms separately, in Lemmas 4.1, 4.2 and 4.4 below.

Lemma 4.1 (Bounds on $\left.\rho^{(N)}\right)$ For $N=1, \rho^{(1)} \leq d^{-2} \beta \mathcal{E}_{0}(d)$, and, for $N \geq 2$,

$$
\rho^{(N)} \leq \beta^{N} \frac{G_{d-1} \mathcal{E}_{1}(d)}{d^{2}(d-1)} a_{d}^{N-2} .
$$

Proof This is exactly the same as the proof of Proposition 3.2 except that at the very last step we use

$$
\sum_{\omega_{1}^{(0)}} \frac{1}{2 d} I_{\left\{\omega_{1}^{(0)}= \pm e_{1}\right\}}=\frac{1}{d} .
$$

Lemma 4.2 (Bounds on $\left.\chi^{(N)}\right)$ For $N=1, \chi^{(1)} \leq d^{-1} \mathcal{E}_{0}(d)$, and, for $N \geq 2$,

$$
\chi^{(N)} \leq N \beta^{N-1} \frac{G_{d-1} \mathcal{E}_{1}(d)}{d(d-1)} a_{d}^{N-2} .
$$


Proof Proceeding exactly as in the proof of Proposition 3.2, except that the bound on $\left|\Delta_{k}\right|$ is missing the $\beta$ term, we obtain $\chi_{k}^{(1)} \leq d^{-1} \mathcal{E}_{0}(d)$ and, for $N \geq 2$,

$$
\chi_{k}^{(N)} \leq \beta^{N-1} d^{-1}(d-1)^{-1} G_{d-1} \mathcal{E}_{1}(d) a_{d}^{N-2} .
$$

The resulting bound on $\chi^{(N)}$, (which is simply $\beta^{-1} N$ times (3.10)) is then easily obtained by summing over $k$ from 1 to $N$.

Before proceeding to the bound on $\gamma^{(N)}$, we first need a new lemma similar to Lemma 3.1.

Lemma 4.3 Let $\mathbb{Q}^{\leftrightarrow}, \vec{\eta}_{s}$ denote the law of a self-interacting random walk $\vec{\omega}$ with history $\vec{\eta}_{s}$, where the transition probabilities are those of an ERW with history $\vec{\eta}_{s}$, except that

$$
\mathbb{Q}^{\leftrightarrow}, \vec{\eta}_{s}\left(\omega_{l+1}=\omega_{l}+e_{1} \mid \vec{\omega}_{l}\right)=\mathbb{Q}^{\leftrightarrow_{l}, \vec{\eta}_{s}}\left(\omega_{l+1}=\omega_{l}-e_{1} \mid \vec{\omega}_{l}\right)=\frac{1}{2} .
$$

Then, for all $i \geq 0$,

$$
\sum_{j=1}^{\infty} \frac{(j+i) !}{j !} \sum_{l=0}^{j-1} \mathbb{Q}^{\leftrightarrow,}, \vec{\eta}_{s}\left(\omega_{j}=u\right) \leq(i+1) !\left(\frac{d}{d-1}\right)^{i+2} G_{d-1}^{*(i+2)}
$$

Proof Since one of the $j$ steps is a simple random walk step in the first coordinate, the number of steps in the other coordinates has a $\operatorname{Binomial}\left(j-1, \frac{d-1}{d}\right)$ distribution. Thus,

$$
\begin{aligned}
& \sum_{j=1}^{\infty} \frac{(j+i) !}{j !} \sum_{l=0}^{j-1} \mathbb{Q}^{\leftrightarrow l,} \vec{\eta}_{s}\left(\omega_{j}=u\right) \\
& \quad \leq \sup _{v \in \mathbb{Z}^{d-1}} \sum_{j=1}^{\infty} \sum_{l=1}^{j} \sum_{k=0}^{j-1} \frac{(j+i) !}{j !} \mathcal{P}\left(\mathcal{N}_{j-1}=k\right) \mathbb{P}_{d-1}\left(\omega_{k}=v\right) \\
& =\sup _{v \in \mathbb{Z}^{d-1}} \sum_{k=0}^{\infty} \mathbb{P}_{d-1}\left(\omega_{k}=v\right) \sum_{j=k+1}^{\infty} j \frac{(j+i) !}{j !} \mathcal{P}\left(\mathcal{N}_{j-1}=k\right) \\
& =\sup _{v \in \mathbb{Z}^{d-1}} \sum_{k=0}^{\infty} \mathbb{P}_{d-1}\left(\omega_{k}=v\right) \sum_{r=k}^{\infty} \frac{(r+i+1) !}{r !} \mathcal{P}\left(\mathcal{N}_{r}=k\right) .
\end{aligned}
$$

Now proceed as in the proof of Lemma 3.1 to obtain the result.

Define

$$
\epsilon(d)=\frac{2 d}{(d-1)^{4}} G_{d-1} G_{d-1}^{* 3}+\frac{\mathcal{E}_{1}(d)}{d(d-1)^{2}} G_{d-1}^{* 2} .
$$


Lemma 4.4 (Bounds on $\left.\gamma^{(N)}\right)$ For $N=1,2, \gamma^{(1)} \leq \beta(d-1)^{-2} G_{d-1}^{* 2}, \gamma^{(2)} \leq \beta^{2} \epsilon(d)$ and, for all $N \geq 3$,

$$
\gamma^{(N)} \leq \epsilon(d) \beta^{2}\left(\beta a_{d}\right)^{N-2}+(N-2) \frac{2 \beta^{3} \mathcal{E}_{1}(d)}{(d-1)^{4}} G_{d-1} G_{d-1}^{* 3}\left(\beta a_{d}\right)^{N-3} .
$$

Proof We proceed as in the proof of Proposition 3.2 except that from the definition of $\gamma_{k}^{(N)}$, the product of transition probabilities inside the sum over $j_{k}$ in (3.18), is replaced with (4.6). We use Lemma 4.3 instead of Lemma 3.1 to bound this sum.

When $N=1$, then also $k=1$ and $\gamma_{1}^{(1)}$ is

$$
\begin{aligned}
& \sum_{\omega_{1}^{(0)}} p_{\beta}\left(\omega_{1}^{(0)}\right) \sum_{j_{1}=1}^{\infty} \sum_{l=0}^{j_{1}-1} \sum_{\vec{\omega}_{j_{1}+1}^{(1)}}\left|\Delta_{1}\right| \frac{I_{\left\{\omega_{l+1}^{(1)}-\omega_{l}^{(1)}= \pm e_{1}\right\}}}{2 d} \prod_{\substack{i=0 \\
i \neq l}}^{j_{1}-1} p^{\vec{\omega}_{1}^{(0)} \circ \vec{\omega}_{i}^{(1)}}\left(\omega_{i}^{(1)}, \omega_{i+1}^{(1)}\right) \\
& \leq \frac{\beta}{d^{2}} \sum_{\omega_{1}^{(0)}} p_{\beta}\left(\omega_{1}^{(0)}\right) \sum_{j_{1}=1}^{\infty} \sum_{l=0}^{j_{1}-1} \sum_{\vec{\omega}_{j_{1}}^{(1)}} I_{\left\{\omega_{j_{1}}^{(1)}=\omega_{0}^{(0)}\right\}} \frac{I_{\left\{\omega_{l+1}^{(1)}-\omega_{l}^{(1)}= \pm e_{1}\right\}}}{2} \\
& \quad \times \prod_{\substack{i=0 \\
i \neq l}}^{j_{1}-1} p^{\vec{\omega}_{1}^{(0)} \circ \vec{\omega}_{i}^{(1)}}\left(\omega_{i}^{(1)}, \omega_{i+1}^{(1)}\right)
\end{aligned}
$$

where we have used the usual bound (2.5) and the fact that $\sum_{\omega_{j_{1}+1}^{(1)}} I_{\left\{\omega_{j_{1}+1}^{(1)}=\omega_{j_{1}}^{(1)} \pm e_{1}\right\}}=2$. Now observe that

$$
\frac{I_{\left\{\omega_{l+1}^{(1)}-\omega_{l}^{(1)}= \pm e_{1}\right\}}}{2} \prod_{\substack{i=0 \\ i \neq l}}^{j_{1}-1} p^{\vec{\omega}_{1}^{(0)} \circ \vec{\omega}_{i}^{(1)}}\left(\omega_{i}^{(1)}, \omega_{i+1}^{(1)}\right)=\mathbb{Q}^{\leftrightarrow l,} \vec{\omega}_{1}^{(0)}\left(\vec{\omega}_{j_{1}}=\vec{\omega}_{j_{1}}^{(1)}\right),
$$

so that (4.17) is equal to

$$
\begin{aligned}
& \frac{\beta}{d^{2}} \sum_{\omega_{1}^{(0)}} p_{\beta}\left(\omega_{1}^{(0)}\right) \sum_{j_{1}=1}^{\infty} \sum_{l=0}^{j_{1}-1} \sum_{\vec{\omega}_{j_{1}}^{(1)}} I_{\left\{\omega_{j_{1}}^{(1)}=\omega_{0}^{(0)}\right\}} \mathbb{Q}^{\leftrightarrow_{l}, \vec{\omega}_{1}^{(0)}}\left(\vec{\omega}_{j_{1}}=\vec{\omega}_{j_{1}}^{(1)}\right) \\
& =\frac{\beta}{d^{2}} \sum_{\omega_{1}^{(0)}} p_{\beta}\left(\omega_{1}^{(0)}\right) \sum_{j_{1}=1}^{\infty} \sum_{l=0}^{j_{1}-1} \mathbb{Q}^{\leftrightarrow, \vec{\omega}_{1}^{(0)}}\left(\omega_{j_{1}}=\omega_{0}^{(0)}\right) .
\end{aligned}
$$

Now use (4.13) with $i=0$ to get the required bound.

For the remaining cases we begin by adjusting the sum over $j_{k}$ in (3.18) as in (4.17) and (4.18). When $N>1$ and $k=1$ we use the same bounds as in the proof of Proposition 3.2 except that we use (4.13) with $i=1$ on the sum over $j_{1}$. This gives us a bound on $\gamma_{1}^{(N)}($ when $N>1$ ) of 


$$
\frac{2 \beta}{d^{2}}\left(\frac{d}{d-1}\right)^{3} G_{d-1}^{* 3} \frac{\beta}{d-1} G_{d-1} \prod_{i=2}^{N-1} \beta a_{d}
$$

When $N>1$ and $k=N$, we use the same bounds as in the proof of Proposition 3.2 except that we use (4.13) with $i=0$ on the sum over $j_{N}$ in (3.18). This gives us a bound on $\gamma_{N}^{(N)}($ when $N>1$ ) of

$$
\frac{\beta}{(d-1)^{2}} G_{d-1}^{* 2} \frac{\beta}{d} \mathcal{E}_{1}(d) \prod_{i=2}^{N-1} \beta a_{d} .
$$

Similarly when $N>1$ and $1 \neq k \neq N$ (so $N>2$ ) we use (4.13) on the sum over $j_{k}$ in (3.18) to get a bound on $\gamma_{k}^{(N)}$ of the form

$$
\frac{\beta}{d-1} G_{d-1} \frac{\beta}{d} \mathcal{E}_{1}(d) \frac{2 \beta}{d^{2}}\left(\frac{d}{d-1}\right)^{3} G_{d-1}^{* 3} \prod_{\substack{i=2 \\ i \neq k}}^{N-1} \beta a_{d}
$$

Simplifying these expressions and summing over $k$ completes the proof of the lemma.

Corollary 4.5 (Summary of bounds) For all $\beta \in[0,1]$, and $d$ such that $a_{d}<1$

$$
\begin{aligned}
d \sum_{N=1}^{\infty} \rho^{(N)} & \leq \frac{\mathcal{E}_{0}(d)}{d}+\frac{G_{d-1} \mathcal{E}_{1}(d)}{d(d-1)\left(1-a_{d}\right)}, \\
d \sum_{N=1}^{\infty} \chi^{(N)} & \leq \mathcal{E}_{0}(d)+\frac{G_{d-1} \mathcal{E}_{1}(d)\left(2-a_{d}\right)}{(d-1)\left(1-a_{d}\right)^{2}}, \\
d \sum_{N=1}^{\infty} \gamma^{(N)} & \leq \frac{d G_{d-1}^{* 2}}{(d-1)^{2}}+\frac{\epsilon(d) d}{1-a_{d}}+\frac{2 d \mathcal{E}_{1}(d) G_{d-1} G_{d-1}^{* 3}}{(d-1)^{4}\left(1-a_{d}\right)^{2}} .
\end{aligned}
$$

Proof Firstly note that the condition on $a_{d}$ ensures that $\rho^{(N)}, \chi^{(N)}$ and $\gamma^{(N)}$ are all summable over $N$, and in all cases the supremum over $\beta$ occurs at $\beta=1$ (see Lemmas 4.1, 4.2 and 4.4). The results are then easily obtained by summing each of the bounds in Lemmas 4.1, 4.2 and 4.4 over $N$.

\section{Proof of Theorem 1.1}

For $d$ such that $a_{d}<1$, the bounds of Corollary 4.5 hold. From (4.7) we have the required absolute summability conditions in the discussion after (2.12), and in particular (2.12) holds for all $\beta \in[0,1]$. To complete the proof of the theorem, it remains to show that the right hand side of (2.13) is no more than $d^{-1}$. By (4.7) and Corollary 4.5, we have bounded $d$ times the right hand side of (2.13) by the sum of the right hand 
sides of the bounds in Corollary 4.5. Since these terms all involve simple random walk Green's functions quantities, we will need to use estimates of these quantities.

In order to bound $\mathcal{E}_{i}(d)$, we shall first prove that, for all $i \geq 0$,

$$
\mathcal{E}_{i}(d)=\left(\frac{d}{d-1}\right)^{i+1} G_{d-1}^{*(i+1)}-1
$$

In order to prove (5.1), we first make use of [9, Lemma B.3], which states that $G_{d}^{* n}(x)$ is non-increasing in $\left|x_{i}\right|$ for every $i=1, \ldots, d$, so that the supremum in (3.3) can be restricted to $v=0$ and $v=e$ for any neighbour $e$ of the origin. In order to bound $G_{d}^{* n}(e)$, we make use of the fact that for any function $x \mapsto f(x)$ for which $f(e)$ is constant for all $e \in \mathbb{Z}^{d}$ with $|e|=1$, we have $f(e)=\left(D_{d} * f\right)(0)$, so that

$$
\mathcal{E}_{i}(d)=\max \left\{\left(\frac{d}{d-1}\right)^{i+1} G_{d-1}^{*(i+1)}(0)-1,\left(\frac{d}{d-1}\right)^{i+1}\left(D_{d-1} * G_{d-1}^{*(i+1)}\right)(0)\right\}
$$

Note that since $G_{d}(x)=\delta_{0, x}+\left(D_{d} * G_{d}\right)(x) \geq \delta_{0, x}$, we have that $G_{d}^{* i}(0) \geq 1$ and $G_{d}^{*(i+1)}(0)=G_{d}^{* i}(0)+\left(D_{d} * G_{d}^{*(i+1)}\right)(0)$. Therefore,

$$
\begin{aligned}
\left(\frac{d}{d-1}\right)^{i+1} G_{d-1}^{*(i+1)}(0)-1= & \left(\frac{d}{d-1}\right)^{i+1}\left(D_{d-1} * G_{d-1}^{*(i+1)}\right)(0) \\
& +\left(\frac{d}{d-1}\right)^{i+1} G_{d}^{* i}(0)-1
\end{aligned}
$$

which is strictly larger than $\left(\frac{d}{d-1}\right)^{i+1}\left(D_{d-1} * G_{d-1}^{*(i+1)}\right)(0)$ and thus proves (5.1).

By [9, Lemma C.1], $d \mapsto G_{d}^{* n}$ is monotone decreasing in $d$ for each $n \geq 1$, so that it suffices to show that the sum of terms on the right hand sides of (4.22)-(4.24) is bounded by 1 for $d=9$. For this we use the following rigorous Green's functions estimates $[8,9]$ for $d=8$ :

$$
G_{d} \leq 1.07865, \quad G_{d}^{* 2} \leq 1.2891, \quad G_{d}^{* 3} \leq 1.8316
$$

Putting in these values for $d-1=8$ we get that the sum of the right hand sides of the bounds in Corollary 4.5 is at most 0.97 , whence the result follows for $d \geq 9$.

To prove monotonicity for $\beta \in\left[0, \beta_{0}\right]$ for some $\beta_{0}(d)$ for each $d \geq 8$, it is sufficient to prove that $\chi^{(1)}<d^{-1}$ when $d \geq 8$ (and that the other terms are bounded), since this is the only term that does not contain a factor $\beta$ that can be made arbitrarily small by choosing $\beta_{0}$ small. Since $\chi^{(1)} \leq d^{-1} \mathcal{E}_{0}(d)$, it is enough to show that $\mathcal{E}_{0}(d)<1$ for $d=8$, since the right hand sides of (4.22)-(4.24) are bounded for $d \geq 8$. From [9] we have $\frac{6}{5} G_{5}-1<\frac{6}{5}(1.157)-1<1$, and since $\mathcal{E}_{0}(d)$ is decreasing in $d$, this completes the result. 
Acknowledgments The work of RvdH and MH was supported in part by Netherlands Organisation for Scientific Research (NWO). The work of MH was also supported by a FRDF grant from the University of Auckland. The authors thank Itai Benjamini for suggesting this problem to us, Takashi Hara for providing the Green's functions upper bounds in (5.4), and an anonymous referee for many helpful suggestions that significantly improved the presentation of this paper.

Open Access This article is distributed under the terms of the Creative Commons Attribution Noncommercial License which permits any noncommercial use, distribution, and reproduction in any medium, provided the original author(s) and source are credited.

\section{References}

1. Antal, T., Redner, S.: The excited random walk in one dimension. J. Phys. A Math. Gen. 38, 25552577 (2005)

2. Basdevant, A.-L., Singh, A.: On the speed of a cookie random walk. Probab. Theory Relat. Fields. 141(3-4), 625-645 (2008)

3. Basdevant, A.-L., Singh, A.: Rate of growth of a transient cookie random walk. Electr. J. Probab. 13, 811-851 (2008)

4. Benjamini, I., Wilson, D.B.: Excited random walk. Electron. Comm. Probab. 8, 86-92 (2003) (electronic)

5. Bérard, J., Ramírez, A.: Central limit theorem for excited random walk in dimension $d \geq 2$. Electr. Comm. Probab. 12, 300-314 (2007)

6. Bolthausen, E., Sznitman, A.-S., Zeitouni, O.: Cut points and diffusive random walks in random environment. Ann. Inst. H. Poincaré Probab. Stat. 39(3), 527-555 (2003)

7. Davis, B.: Brownian motion and random walk perturbed at extrema. Probab. Theory Relat. Fields. 113, 501-518 (1999)

8. Hara, T.: Private communication (2007)

9. Hara, T., Slade, G.: The lace expansion for self-avoiding walk in five or more dimensions. Rev. Math. Phys. 4, 235-327 (1992)

10. Holmes, M.: A monotonicity property for a random walk in a partially random environment (2008, preprint)

11. Holmes, M.: Excited against the tide: a random walk with competing drifts (2008, preprint)

12. Kozma, G.: Excited random walk in three dimensions has positive speed (2003, unpublished)

13. Kozma, G.: Excited random walk in two dimensions has linear speed (2005). (arXiv:math/0512535v1 [math.PR])

14. Sznitman, A.-S., Zerner, M.: A law of large numbers for random walks in random environment. Ann. Probab. 27, 1851-1869 (1999)

15. van der Hofstad, R., Holmes, M.: An expansion for self-interacting random walks (2007). (arXiv: 0706.0614v2 [math.PR])

16. Zerner, M.: Multi-excited random walks on integers. Probab. Theory Relat. Fields. 133, 98-122 (2005) 